This method is excellent for mycelium and thickwalled spores; but large thin-walled spores are apt to collapse as a result of the rather drastic treatment. As Armitage ${ }^{1}$ points out, the chlorazol black stains more brilliantly if methyl alcohol is used as solvent rather than ethyl alcohol.

The thickness of the agar included in the preparation is sometimes a source of trouble in critical observation with high-aperture objectives. To avoid this, the following technique may be used which, though slower, gives beautiful preparations. Blocks of agar bearing the fungus, about $4 \mathrm{~mm}$. square, are cut out and fixed in the following mixture: commercial formalin, $10 \mathrm{ml}$; ; glacial acetic acid, $5 \mathrm{ml}$; distilled water, $85 \mathrm{ml}$. For this formula we are indebted to Dr. B. Barnes. Material is fixed for at least $24 \mathrm{hr}$., but may be left in the fixative indefinitely without harm. The agar block is washed until it no longer smells of acetic acid, and then stuck, fungus downwards, on a No. 1 cover slip by means of Szombathy's gelatine, as recommended by Chamberlain ${ }^{2}$. The agar is allowed to dry in a warm place (not above $35^{\circ} \mathrm{C}$.) until it begins to shrink; the surface bearing the fungus is prevented from shrinking by adhesion to the glass. When the agar is firmly attachod to the cover slip, the block is sliced away with a sharp razor blade as close to the glass as possible. The material, still on the glass, is then stained with Heidenhain's hæmatoxylin, dehydrated by passage through a gradually ascending series of alcohols, cleared with terpineol and mounted in canada balsam. If desired, clearing may be omitted and 'Euparal' used as a mountant following absolute alcohol.

In this technique, any suitable stain may be used, but once alcohol has been applied to the preparation it is inadvisable to return to water, as swelling of the agar may cause it to become detached from the glass.

Details of these and other techniques will be given elsewhere.

Biological Laboratories,

C. L. Duddington Sylvia M. Dixon

The Polytechnic,

Regent Street,

London, W.1.

Feb. 22.

${ }^{1}$ Armitage, F. D., Trans. Brit. Mycol. Soc., 27, 131 (1944),

"Chamberlain, C. J., "Methods in Plant Histology"' (Chicago, 1932).

\section{Corpora amylacea in the Central Nervous System}

IT has long been known that small round corpuscles may be found around the blood vessels of the central nervous system in subjects of advanced age. As the staining properties of those bodies are similar to those of starch, Virchow called them corpora amylacea. Several authors have examined them in attempts to determine their origin, but without definite result. The present contribution describes an attempt to define the morphology and some of the chemical properties of corpora amylacea, using the polarizing microscope as well as the periodic acid-leucofuchsin method ${ }^{1}$.

Under the polarizing microscope, the round bodies show pronounced double refraction with a positive Maltese cross (Fig. 1), the path-difference being
$15-20 \mu$ in our sections. This finding does not agree with those previously given in the literature ${ }^{2}$. The character of the double refraction does not change in the imbibition test, suggesting that self-birefringent lipids may be considered as the principal constituents of these bodies.

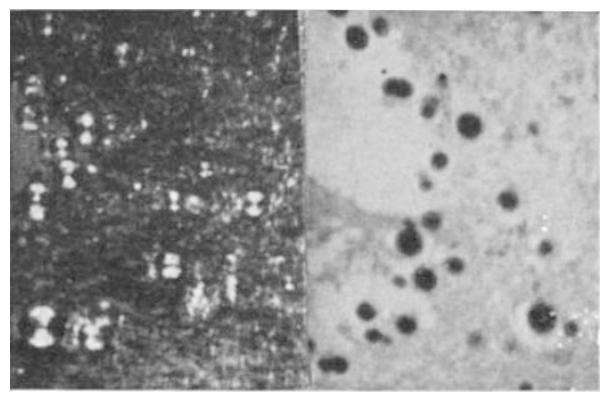

(1)

(2)

Sections of corpora amylacea : (1) polarized light, crossed nicols and Senarmont compensation $(\times 80) ;(2)$ periodic acid-leucofuchsin method $(\times 80)$

The myelin sheath gives a positive aldehydic reaction after treatment with periodic acid; the round bodies were treated similarly in order to obtain information about the possibility of a genetical relationship between them and the myelin sheath. Frozen or paraffin-embedded sections of corpora smylacea showed a brilliant rose-purple colour (Fig. 2) ; myelin sheath, however, gave a positive result only in frozen sections. In the case of corpora amylacea, the reaction was not inhibited if the bodies were extracted with solvents which would remove cerebrosides. This is probably due to the fact that the cerebrosides which are responsible for the aldehyde reaction are linked to proteins, thereby becoming unextractable ${ }^{3}$.

Summarizing the above results, it is suggested that the principal constituents of corpora amylacea are lipids, and that their intense aldehydic reaction after treatment with periodic acid indicates that they possibly belong to the carbohydrate-containing cerebrosides. It well may be that the origin of corpora amylacea is in the cerebrosides of the degenerated myelin sheath.

\section{J. Molnar}

Department of Pathology,

Municipal Hospital,

Szombathely, Hungary. Feb. 26.

'McManus, J. F. A., Nature, 158, 202 (1946).

? Pullinger, B. D., J. Path. Bact., 55, 99 (1943).

8 Morrison, R. W., and Hack, M. H., Amer. J. Path., 25, 597 (1949).

\section{Nuclear Reaction Radiography}

Autoradiography has been employed for many years for the purpose of determining the distribution of an element in a given material. The ossential requirement for the use of this method is that the element in question itself should emit a radiation which can be registered on a photographic film pressed against the surface of the sample. To make an element betray its presence in this way, one provides a certain fraction of marked atoms by adding a small amount of radioactive isotopes of the same element. 